\title{
Insula and Orbitofrontal Cortical Morphology in Substance Dependence Is Modulated by Sex
}

\author{
J. Tanabe, P. York, T. Krmpotich, D. Miller, M. Dalwani, J.T. Sakai, S.K. Mikulich-Gilbertson,
}

L. Thompson, E. Claus, M. Banich, and D.C. Rojas

\begin{abstract}
BACKGROUND AND PURPOSE: Frontolimbic circuits are involved in learning and decision-making processes thought to be affected in substance-dependent individuals. We investigated frontolimbic cortical morphometry in substance-dependent men and women and determined whether morphometric measurements correlated with decision-making performance.
\end{abstract}

MATERIALS AND METHODS: Twenty-eight abstinent SDI (17 men/11 women) were compared with 28 controls (13 men/15 women). Cortical thicknesses and volumes were computed by using FreeSurfer. After controlling for age and intracranial volume, group and sex effects were analyzed in 3 a priori regions of interest: the insula, orbitofrontal cortex, and anterior cingulate cortex by using analysis of covariance. A secondary whole-brain analysis was conducted to verify region-of-interest results and to explore potential differences in other brain regions.

RESULTS: Region-of-interest analyses revealed a main effect of group on the left insula cortex, which was thinner in SDI compared with controls $(P=.02)$. There was a group by sex interaction on bilateral insula volume (left, $P=.02$; right, $P=.001)$ and right insula cortical thickness $(P=.007)$. Compared with same-sex controls, female SDI had smaller insulae, whereas male SDI had larger insulae. Neither ACC nor OFC significantly differed across group. Performance on a decision-making task was better in controls than SDI and correlated with OFC measurements in the controls.

CONCLUSIONS: SDI and controls differed in insula morphology, and those differences were modulated by sex. No group differences in OFC were observed, but OFC measurements correlated with negative-reinforcement learning in controls. These preliminary results are consistent with a hypothesis that frontolimbic pathways may be involved in behaviors related to substance dependence.

ABBREVIATIONS: ACC = anterior cingulate cortex; DSM-IV = Diagnostic and Statistical Manual of Mental Disorders-Fourth Edition; OFC = orbitofrontal cortex; $\mathrm{SDI}=$ substance-dependent individuals; $\mathrm{VBM}=$ voxel-based morphometry

E xposure to drugs of abuse is associated with neural adaptations thought to be important in processing motivations, decisions, and learned associations that may perpetuate drug-taking behavior. ${ }^{1,2}$ Structural neuroimaging studies in drug abuse have focused on the prefrontal cortex and, in particular, the orbitofrontal

Received July 23, 2012; accepted July 30.

From the Departments of Radiology (J.T., P.Y., T.K., D.M.) and Psychiatry (I.T., M.D., J.T.S., S.K.M.-G., L.T., M.B., D.C.R.), University of Colorado School of Medicine, Denver, Colorado; Department of Psychology (M.B.), University of Colorado Boulder, Boulder, Colorado; and The Mind Research Network (E.C.), Albuquerque, New Mexico.

This work was funded by the National Institutes of Health (DA024104, 027748 , 009842, and 011015) and the Kane Family Foundation.

Paper previously presented at: ASNR 50th Annual Meeting and the Foundation of the ASNR Symposium, April 21-26, 2012; New York, New York.

Please address correspondence to Jody Tanabe, MD, 12700 E 17th Ave, Mailstop C278, Aurora, CO 80045; e-mail: jody.tanabe@ucdenver.edu

-- Indicates open access to non-subscribers at www.ajnr.org

http://dx.doi.org/10.3174/ajnr.A3347 cortex, because of its putative role in decision-making related to drug addiction. ${ }^{1,3,4}$ Compared with the prefrontal cortex, less attention has been given to the limbic system, though there is evidence that the insula, a phylogenetically old area and part of the limbic system, may also be involved in addiction. Insula lesions disrupt smoking behavior. ${ }^{5}$ Animal studies have shown that the insula is involved in learning to associate external cues with the rewarding effects of drugs. ${ }^{6}$ Neuroimaging studies suggest that the insula is involved in anxiety, ${ }^{7}$ avoidance learning, ${ }^{8}$ and drug cravings. ${ }^{5}$ It has been hypothesized that the anticipation of drug withdrawal or negative bodily states trigger interoceptive signals in the insula. ${ }^{9-11}$ From there, signals are transmitted to the OFC where information is maintained on-line to inform decisions and guide actions. Few structural imaging studies of substance dependence, however, have focused on the insula.

Structural imaging of substance-dependent populations has demonstrated reduced gray matter volume in the OFC, anterior cingulate, and dorsolateral prefrontal cortex, ${ }^{12-17}$ but the results 
have not been entirely consistent. No difference in cortical volume was observed in a study of 16 drug users ${ }^{18}$ or in another study of 34 cocaine-dependent subjects, ${ }^{19}$ compared with controls. One possibility for equivocal results may be methodologic. Many prior studies used voxel-based morphometry, which involves voxelwise tissue classification, normalization to a standard atlas, and statistical comparison to determine differences in cortical volume. Volume represents 1 dimension of cortical macrostructure, however, and VBM does not account for the complex folding patterns of the cortex or variations in columnar architecture. In contrast to VBM, surface-based algorithms model sulcal and gyral topologies and provide measures of cortical thickness. ${ }^{20}$ Using such surfacebased modeling, Makris et $\mathrm{al}^{21}$ have shown cortical thinning in cocaine-dependent subjects in a reward network comprising the orbitofrontal, insula, cingulate, and dorsolateral prefrontal cortices. Durazzo et $\mathrm{al}^{22}$ demonstrated cortical thinning in similar brain regions in alcoholics compared with controls. Thompson et $\mathrm{al}^{17}$ found decreased gray matter in the limbic system of methamphetamine users, and Kühn et $\mathrm{al}^{23}$ showed thinning of the medial OFC in smokers compared with nonsmokers.

Another possible reason for inconsistent results is that most brain morphometry studies focus on male substance users, yet evidence suggests that sex is an important modulator of drugrelated behavior, brain structure, and function. ${ }^{24,25}$ Sex differences in cortical thickness have been observed in healthy controls. ${ }^{26}$ Alterations in brain structure and function differ in female compared with male substance users. For example, Medina et $\mathrm{al}^{27}$ found that compared with same-sex controls, prefrontal cortex volume was lower in young female alcohol users, while it was larger in young male alcohol users. Male and female cocaine users show different responses to cocaine cues. ${ }^{25}$ Men and women are also known to differ in their vulnerability and treatment response to drugs and alcohol. ${ }^{24}$

The goal of this study was to evaluate frontolimbic cortical morphology in substance-dependent individuals. We hypothesized that SDI compared with controls would have significantly lower mean cortical thickness and volume in the insula, OFC, and ACC. Second, we investigated whether drug-associated cortical morphometry was modulated by sex. To explore the potential significance of these changes, we correlated morphometry with behavioral measures and drug use.

\section{MATERIALS AND METHODS \\ Subjects}

Twenty-eight substance-dependent individuals and 28 controls were studied. Table 1 shows demographics. Behavioral data have been previously reported on a majority of these subjects. ${ }^{28}$ SDI with DSM-IV stimulant dependence were recruited from sex-specific long-term residential drug-treatment programs. Drug characteristics are shown in Table 2. Participants are referred to the treatment programs from the criminal justice system. They typically enroll in our study after 1-2 months in treatment. Abstinence from drugs is monitored by observation and random urine screening while participants are in treatment. Across drugs, the duration of abstinence is relatively long (mean, $1.46 \pm 1.02$ years; median, 1.00 year; range, 1 month to 3 years).

Controls were recruited from the community and were ex- cluded if they met DSM-IV criteria for lifetime dependence on alcohol or any drugs except tobacco. All subjects were excluded for a history of head trauma with loss of consciousness exceeding 15 minutes, neurologic disease, schizophrenia, bipolar disorder, or major depression in the past 2 months.

All subjects provided written informed consent approved by the institutional review board and then completed structured diagnostic interviews, an intelligence quotient test, a reinforcement learning task, and the Wisconsin Card Sorting Test, which were administered by a trained research assistant.

\section{Diagnostic and Structured Interviews}

Composite International Diagnostic Interview: Substance Abuse Module. Results from this computerized structured interview characterized lifetime substance dependence diagnoses in SDI and ensured that controls did not meet criteria for dependence by providing DSM-IV diagnoses for 11 substances: amphetamine, cocaine, marijuana, alcohol, nicotine, hallucinogens, opioids, inhalants, sedatives, club drugs, and phencyclidine.

Diagnostic Interview Schedule, Version I. This computerized structured interview was administered to exclude subjects with schizophrenia, bipolar disorder, or current (within 2 months) major depression.

IQ. The Wechsler Abbreviated Scale of Intelligence 2, subtest version (Vocabulary and Matrix Reasoning) was used to estimate general intelligence.

Decision-Making Task. Negative-reinforcement learning was measured by using a modification of the Iowa Gambling Task. ${ }^{29}$ The task is a behavioral test of decision-making and has been validated in individuals with substance dependence. ${ }^{30,31}$ The task assesses whether a subject learns, with time, to Play or Pass on each of 4 "decks" of cards, to maximize a hypothetic monetary outcome. Played during the long run, 2 decks result in a net gain (advantageous), and 2, in a net loss (disadvantageous). The subject must learn to "Pass" disadvantageous and "Play" advantageous decks. The number of Pass responses is calculated for the first half (time-1) and second half (time-2) of the task. Negative-reinforcement learning occurs when the number of "Passes" on disadvantageous decks increases with time. This study used a variant of the Iowa Gambling Task that is sensitive to differences in negative-reinforcement learning in SDI, and the differences are driven by decreased ability to avoid outcomes associated with large, as opposed to frequent, losses in SDI. ${ }^{28}$

Wisconsin Card Sorting Test. All subjects completed this standardized measure of general executive function. The dependent variable was the number of perseverative errors.

MR Imaging. Images were acquired on a 3T Signa MR imaging (GE Healthcare, Milwaukee, Wisconsin) scanner by using a standard quadrature head coil. A high-resolution 3D T1weighted spoiled gradient recalled-echo inversion recovery sequence was performed with the following parameters: $\mathrm{TR}=$ $45, \mathrm{TE}=20$, flip angle $=45^{\circ}$, matrix $=256^{2} ; \mathrm{FOV}=240 \mathrm{~mm}^{2}$ $\left(0.9 \times 0.9 \mathrm{~mm}^{2}\right.$ in-plane $)$; section thickness $=1.7 \mathrm{~mm}$, coronal

AJNR Am J Neuroradiol 34:1150-56 Jun 2013 www.ajnr.org 1151 
plane. A neuroradiologist evaluated all MR imaging scans for abnormalities.

Image Processing. Image processing was conducted by using FreeSurfer (Version 4.5.0), an automated program for calculating cortical thickness (http://surfer.nmr.mgh.harvard.edu). Cortical surfaces were reconstructed by running several preprocessing steps that included intensity normalization, skull stripping, generation of a pial surface from the outer edge of the gray matter, and generation of a white/gray matter boundary by using triangular tessellation. The cortical thickness was the estimated distance between the pial surface and the white/gray matter boundary. The resulting surface models for each dataset were inspected by a research assistant blinded to group status. Inaccuracies in the white/gray interface were corrected by using manually placed "control points" to improve identification of missed white matter. Pial surfaces were corrected for erroneous inclusion of the dura or skull. Final edited datasets were evaluated for accuracy by a neuroradiologist who was blinded to group status. Spatial normalization to a gyral-based cortical template resulted in automatic labeling of 34 parcellation units for each hemisphere. ${ }^{32}$ Total intracranial volume was recorded.

\section{Image Analysis}

The primary analysis was a region-of-interest approach based on a priori predictions from the literature of group differences. The secondary analysis was conducted over the whole brain to confirm region-of-interest results and to explore morphometry differences in other brain regions.

Region of Interest. Three ROIs were based on standardized parcellation units in the atlas of Desikan et $\left.\mathrm{al}^{32}: 1\right)$ the orbital frontal cortex consisted of the average of medial orbitofrontal, lateral orbitofrontal, and pars orbitalis parcellation units; 2) the anterior cingulate cortex consisted of the average of rostral and caudal ACC parcellation units; 3) the insula consisted of the insula parcellation unit. FreeSurfer computes volume, cortical thickness, and surface area. Because these measurements are linearly related, we restricted our analyses to volume and cortical thickness, drawing from a previous study showing that these 2 measures are relatively sensitive to group differences. ${ }^{22}$

Whole Brain. Vertex-wise general linear modeling by using analysis of covariance tested for main effects of group and sex and interactions on cortical thickness. Age and intracranial volume were entered as covariates. Data were smoothed with a $10-\mathrm{mm}$ full width at half maximum Gaussian kernel. To verify region-of-interest results, we set statistical maps at a threshold of $P<.001$ uncorrected. For exploratory analyses, maps were set at a voxelwise threshold of $P<.001$, and cluster-level threshold was set at $P<.05$, corrected for multiple comparisons, by using Monte Carlo $Z$ simulation and 5000 iterations.

\section{Statistical Analyses}

We ensured that data were approximately normally distributed, and then we compared groups on continuous and dichotomous demographic variables by using independent $t$ tests and $\chi^{2}$ tests, respectively (Statistical Package for the Social Sciences, PASW 18;
Table 1: Demographic data for SDI and controls ${ }^{\mathrm{a}}$

\begin{tabular}{lcc}
\hline & SDI & Controls \\
\hline Sex & $17 \mathrm{M} / 11 \mathrm{~F}$ & $13 \mathrm{M} / 15 \mathrm{~F}$ \\
Age $(\mathrm{yr})$ & $35.0 \pm 7.4$ & $36.0 \pm 8.6$ \\
Education $^{\mathrm{b}}$ & $12.1 \pm 2.3$ & $13.6 \pm 1.9$ \\
IQ & $102.2 \pm 9.9$ & $100.8 \pm 12.1$ \\
Last drug use $(\mathrm{yr})^{\text {Nat }}$ & $1.46 \pm 1.0$ & \\
\hline
\end{tabular}

Note:- Last drug use indicates drugs and alcohol.

a Data are mean (SD).

${ }^{\mathrm{b}} \mathrm{P}=.01$.

SPSS, Chicago, Illinois). Although we had directional hypotheses, all comparisons used a 2-tailed .05 significance level.

Cortical Morphometry. For each region of interest, cortical thickness and volume were analyzed for main effects of sex and group, and sex by group interactions by using analysis of covariance, after adjusting for age and intracranial volume. Using adjusted means, we calculated effect sizes (Cohen $d$ ). ${ }^{33}$

Negative-Reinforcement Learning. The number of passes on disadvantageous decks at time- 1 and time- 2 was evaluated with a 3-way repeated measures ANOVA with a between-subject effect of group (SDI versus controls), within-subject effects of time and type of feedback (magnitude versus frequency), and all interactions (see Thompson et $\mathrm{al}^{28}$ ). For correlations, the number of passes on disadvantageous decks was summed with time to a single variable.

Wisconsin Card Sorting Test. Groups were compared on the number of perseverative and nonperseverative errors by using an independent $t$ test.

Relationship between Region-of-Interest and Behavioral Variables. Linear associations among negative-reinforcement learning, perseverative errors, IQ, and region-of-interest variables were analyzed with partial correlations, after adjusting for age and intracranial volume. Variables were collapsed over group unless they differed across groups, in which case correlations were conducted separately for controls and SDI.

Relationship between Region-of-Interest and Drug-Use Variables in SDI. Linear associations among duration of stimulant, alcohol, and nicotine dependence; last drug use; and region-of-interest variables were analyzed with partial correlations, after adjusting for age and intracranial volume.

\section{RESULTS}

Subjects

Demographics. There were no significant group differences in sex, age, or IQ. SDI had, on average, 1.5 fewer years of education than controls $(P=.01)$. Table 1 shows demographic variables. Drug characteristics are shown in Table 2. Men and women did not differ in drug characteristics.

\section{Imaging}

Region-of-Interest Cortical Thickness. Analysis of covariance indicated a main effect of group $[F(1,50)=5.42, P=.02]$ on the left insula cortical thickness. The left insula cortex was thinner in SDI than in controls. There was a group by sex interaction on the right 
Table 2: Drug characterization for SDI and controls ${ }^{a}$

\begin{tabular}{|c|c|c|c|c|c|c|}
\hline \multirow[b]{2}{*}{ Drug } & \multicolumn{3}{|c|}{ SDI $(n=28)$} & \multicolumn{3}{|c|}{ Controls $(n=28)$} \\
\hline & No. (\%) & Last Use (yr) & Duration (yr) & No. (\%) & Last Use (yr) & Duration (yr) \\
\hline Stimulants & 28 (100\%) & $2.0 \pm 1.7$ & $8.9 \pm 5.2$ & 0 & 0 & \\
\hline Nicotine & $22(79 \%)$ & $1.3 \pm 4.5$ & $13.3 \pm 8.5$ & $7(25 \%)$ & $4.8 \pm 6.7$ & $12.4 \pm 10.2$ \\
\hline Alcohol & $16(57 \%)$ & $1.9 \pm 2.2$ & $9.2 \pm 6.4$ & 0 & 0 & \\
\hline Heroin & 4 (14\%) & $2.1 \pm 2.6$ & & 0 & 0 & \\
\hline Cannabis & $2(7 \%)$ & $0.5 \pm 0.4$ & & 0 & 0 & \\
\hline
\end{tabular}

${ }^{a}$ Data are mean (SD).

insula cortical thickness $[F(1,50)=8.00, P=.007]$. Compared with sex-matched controls, the right insula cortex was $6.7 \%$ thinner in substance-dependent women and 3\% thicker in substancedependent men. There was no effect of group on ACC or OFC thickness. There was a main effect of sex on OFC thickness (right: $F[1,50]=4.20, P=.05$; left: $F[1,50]=4.09, P=.05)$. Women had thinner OFCs than men. The results are shown in Table 3.

\section{Region-of-Interest Cortical Volume}

Analysis of covariance indicated a group by sex interaction on insula volume (left: $F[1,50]=5.59, P=.022$; right: $F[1,50]=$ $12.26, P=.001)$. Compared with that in sex-matched controls, the left insula cortical volume was $10.6 \%$ smaller in substancedependent women and 6.6\% larger in substance-dependent men. Compared with sex-matched controls, right insula cortical volume was $12.9 \%$ smaller in substance-dependent women and $9.3 \%$ larger in substance-dependent men (Fig 1). There was no main effect of group on ACC or OFC volume. There was a main effect of sex on OFC volume (left: $F[1,50]=4.04, P=.050$; right: $F[1,50]=$ $7.91, P=.007)$. Women had smaller OFC volumes than men. Results are shown in Table 4.

\section{Whole-Brain Analysis}

The exploratory whole-brain analysis revealed no difference in volume or thickness, after correcting for multiple comparisons. Trends in vertex-wise analyses confirmed region-of-interest results. Figure 2 shows an analysis of covariance on cortical thickness for main effects of group, sex, and group-by-sex interactions, at a threshold of $P<$ .001 , uncorrected. Trends for a thinner cortex were observed in the insula and pars opercularis for group (red: SDI < controls); insula and OFC for sex (red: female $<$ male); and insula for interactions (red: female SDI $<$ female controls and male SDI $>$ male controls).

\section{Behavioral Tests}

Negative-Reinforcement Learning. We showed previously that compared with controls, SDI did not learn to Pass on decks asso- ciated with large, as opposed to frequent, losses after adjusting for education, suggesting that differences in negative-reinforcement learning are driven by high-magnitude loss. ${ }^{28}$

Wisconsin Card Sorting Test. There were no group differences in perseverative or nonperseverative errors.

\section{Relationships between Morphometry and Behavior}

In controls, there was a correlation between negative-reinforcement learning and OFC thickness (left: $r=0.41, P=.04$; right: $r=0.58, P=.002$ ) and volume (left: $r=0.10, P=.63$; right: $r=0.52, P=.007)$. Figure 3 shows that learning to avoid disadvantageous decks was associated with thicker OFCs in controls but not in SDI. Men and women did not differ. There were no correlations among negative-reinforcement learning, Wisconsin Card Sorting Test, IQ, and any other region-of-interest measures.

\section{Relationships between Morphometry and Drug Use}

There were negative correlations between the duration of stimulant dependence and left insula cortical thickness $(r=-0.45$, $P=.021)$; duration of nicotine dependence and left OFC volume $(r=-0.46, P=.040)$ and left insula volume $(r=-0.47$, $P=.039)$; and duration of alcohol dependence and left OFC volume $(r=-0.53, P=.051)$. There were no correlations between region-of-interest measures and last drug use.

\section{DISCUSSION}

Substance-dependent individuals had thinning of the left insula cortex compared with controls. Previous studies in substance users have reported cortical thinning in multiple brain regions that included the insula. ${ }^{21,34}$ Makris et $\mathrm{al}^{21}$ found significant cortical thinning in 20 cocaine users compared with controls within a reward network that included the right insula, OFC, cingulate gyrus, and dorsolateral prefrontal cortex. Thinning of both the right and left insula cortices has been reported in adolescent boys who were heavy users of marijuana. ${ }^{34}$ We observed an approxi-

Table 3: Regional cortical thickness ${ }^{a}$

\begin{tabular}{|c|c|c|c|c|c|c|c|c|c|}
\hline & \multicolumn{2}{|c|}{ Controls } & \multicolumn{2}{|c|}{ SDI } & \multicolumn{3}{|c|}{$P$ Value } & \multicolumn{2}{|c|}{ Effect Size } \\
\hline & Male $(n=13)$ & Female $(n=15)$ & Male $(n=17)$ & Female $(n=11)$ & Group & Sex & Interaction & Group & Sex \\
\hline L ACC & $2.72 \pm 0.22$ & $2.57 \pm 0.40$ & $2.67 \pm 0.23$ & $2.65 \pm 0.28$ & .96 & .18 & .45 & 0.01 & 0.42 \\
\hline R ACC & $2.69 \pm 0.17$ & $2.75 \pm 0.23$ & $2.71 \pm 0.26$ & $2.70 \pm 0.23$ & .098 & .049 & .33 & 0.01 & 0.21 \\
\hline L Insula & $2.95 \pm 0.15$ & $2.99 \pm 0.20$ & $2.91 \pm 0.19$ & $2.84 \pm 0.19$ & $.024^{b}$ & .74 & .40 & 0.62 & 0.10 \\
\hline R Insula & $2.97 \pm 0.08$ & $2.99 \pm 0.16$ & $3.04 \pm 0.17$ & $2.79 \pm 0.22$ & .08 & .09 & $.007^{\mathrm{b}}$ & & \\
\hline L OFC & $2.45 \pm 0.08$ & $2.41 \pm 0.17$ & $2.47 \pm 0.15$ & $2.40 \pm 0.10$ & .81 & $.046^{\mathrm{b}}$ & .53 & 0.07 & 0.62 \\
\hline R OFC & $2.46 \pm 0.13$ & $2.39 \pm 0.11$ & $2.49 \pm 0.11$ & $2.41 \pm 0.15$ & .73 & $.053^{\mathrm{b}}$ & .87 & 0.09 & 0.59 \\
\hline
\end{tabular}

Note:- R indicates right; L, left.

a Data are mean (SD). Cortical thickness is in millimeters.

b Significant. 


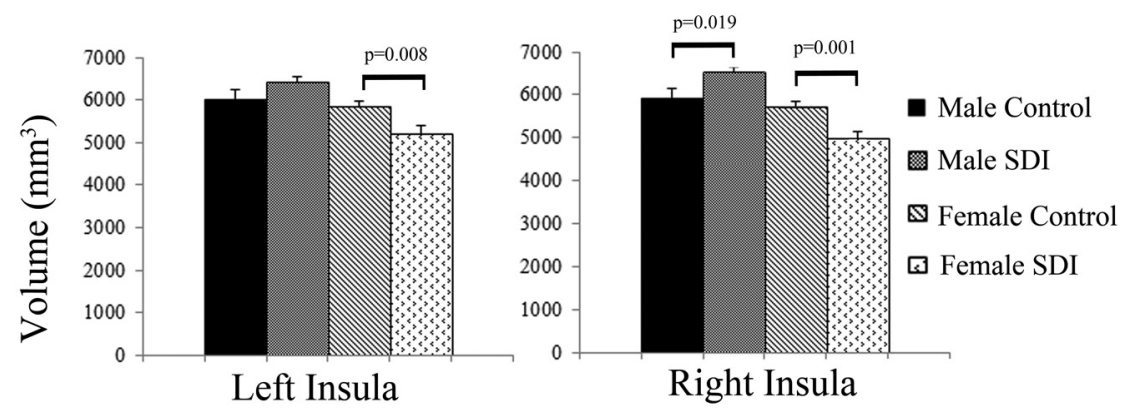

FIG 1. Bar graphs show left and right insula volumes for group and sex. Data are means (SD). related behavior, while an "overactive or larger" insula would be associated with more severe drug-related behavior. Such directionality may be too simplistic, however. Samanez-Larkin et $\mathrm{al}^{8}$ showed that greater, not lower, insula activity predicted better avoidance learning; and Paulus et $\mathrm{al}^{39}$ found that lower, not higher, insula activity during decisionmaking predicted methamphetamine relapse. Thus, the relationship between substance dependence and insula function and structure remains associational, and

mately $3 \%$ reduction in cortical thickness across the insula parcellation unit (Cohen $d=0.62$ ), comparable in magnitude with cortical thickness differences reported in $\mathrm{drug}^{21,23,34}$ and nicotine users. $^{21,23,34}$ Thompson et $\mathrm{al}^{17}$ found lower gray matter concentrations in the limbic system of methamphetamine users, but in the hippocampus and cingulate rather than the insula. VBM studies have also reported reduced bilateral insula gray matter volume in cocaine ${ }^{13}$ and methamphetamine users. ${ }^{35}$ In our study, the time between last drug use and MR imaging ( 1.5 years) was longer than that in prior morphometric studies. Considering that partial recovery of volume has been observed with abstinence from alcohol $^{36}$ and methamphetamine, ${ }^{37}$ the results are unlikely to reflect transient drug effects. There was no correlation between morphometry and abstinence, suggesting that if partial recovery did occur, such changes would have stabilized.

Functional neuroimaging studies have suggested that the insula is involved in cocaine craving, risk-taking, and harmavoidance. ${ }^{8,38}$ The insula is thought to be associated with interoception or the perception of physical states of the body. The insula may process malaise or negative motivational states such as drug withdrawal. The latter is suggested by animal work showing that lithium-induced malaise is blunted in rats following chemical lesioning of the insula. ${ }^{6}$ Alternatively, the insula may signal the urge to take drugs. Naqvi and Bechara ${ }^{11}$ have shown that smokers with brain injuries to the insula have a lower urge to smoke and an easier time quitting compared with smokers with extra-insular brain injuries. ${ }^{5}$ Contreras et $\mathrm{l}^{6}$ have shown that insula lesions in animals result in decreased conditioned place preference to amphetamines, suggesting that the insula is important in learning to pair stimulus cues and reward. The studies by Naqvi and Bechara ${ }^{5}$ and Contreras et $\mathrm{al}^{6}$ might lead one to predict that an "underactive or smaller" insula would be associated with less severe drug- further work is needed to elucidate these mechanisms.

The association between substance dependence and insula morphometry was not the same in men and women. An interaction between sex and group on bilateral insula volume and right insula cortical thickness was unexpected. Compared with sexmatched controls, substance-dependent women had smaller insulsae while substance-dependent men had larger insulae. Medina et $\mathrm{al}^{27}$ observed the same pattern in the prefrontal cortex of youth with alcohol-use disorders. The investigators proposed 2 possibilities: that alcohol may impair normal dendritic pruning in boys or that boys are less sensitive to the known toxic effects of alcohol on myelin. Recently, Potenza et $\mathrm{al}^{40}$ demonstrated sex differences in corticostriatal limbic activity in cocaine users during cue-induced craving. In that study, female cocaine users were sensitive to stress-induced craving and male cocaine users were sensitive to drug-induced craving. Given that the insula is thought to play a role in processing anxiety and negative affective states, one could speculate that the thinner insula in female SDI might be related to more negative affective processing compared with male SDI. Future studies examining sex, affect, and insula morphology and laterality are needed to clarify these relationships.

Contrary to our prediction, we did not observe group differences in volume or thickness in the OFC or anterior cingulate, as others have reported. ${ }^{1,2,41}$ Makris et $\mathrm{al}^{21}$ observed thinning in several sectors of the prefrontal cortex in cocaine users. Lawyer et $\mathrm{al}^{42}$ found no cortical thickness differences in 40 patients dependent on d-amphetamine. As in our study, Lawyer et al used FreeSurfer, which has been shown to have high accuracy, reliability, and precision ${ }^{20,43}$ but may be less sensitive than the Cardviews software (http://www.cma.mgh.harvard.edu/iatr/display.php?spec=id \&ids $=1$ ) used by Makris et $\mathrm{al}^{21}$ that models a slightly more liberal

Table 4: Regional cortical volume ${ }^{\mathrm{a}}$

\begin{tabular}{|c|c|c|c|c|c|c|c|c|c|}
\hline & \multicolumn{2}{|c|}{ Controls } & \multicolumn{2}{|c|}{ SDI } & \multicolumn{3}{|c|}{$P$ Value } & \multicolumn{2}{|c|}{ Effect Size } \\
\hline & Male $(n=13)$ & Female $(n=15)$ & Male $(n=17)$ & Female $(n=11)$ & Group & Sex & Interaction & Group & Sex \\
\hline L ACC & $4276 \pm 1324$ & $3375 \pm 690$ & $4191 \pm 755$ & $3407 \pm 970$ & .40 & .34 & .16 & 0.20 & 0.21 \\
\hline R ACC & $3988 \pm 695$ & $3782 \pm 1014$ & $4069 \pm 873$ & $3574 \pm 721$ & .33 & 1.00 & .81 & 0.27 & 0.00 \\
\hline L Insula & $6009 \pm 797$ & $5844 \pm 522$ & $6431 \pm 440$ & $5222 \pm 577$ & .11 & .15 & $.022^{b}$ & & \\
\hline R Insula & $5924 \pm 783$ & $5722 \pm 449$ & $6528 \pm 542$ & $4982 \pm 557$ & .09 & .07 & $.001^{b}$ & & \\
\hline L OFC & $13659 \pm 1606$ & $12854 \pm 1292$ & $15142 \pm 1486$ & $12547 \pm 1410$ & .36 & $.05^{\mathrm{b}}$ & .18 & 0.25 & 0.61 \\
\hline R OFC & $14511 \pm 1760$ & $13153 \pm 887$ & $15643 \pm 1778$ & $12972 \pm 1219$ & .60 & $.007^{\mathrm{b}}$ & .54 & 0.14 & 0.85 \\
\hline
\end{tabular}

Note:- $\mathrm{R}$ indicates right; L, left.

${ }^{a}$ Data are mean (SD). Cortical volume is in cubic millimeters.

${ }^{\mathrm{b}}$ Significant. 


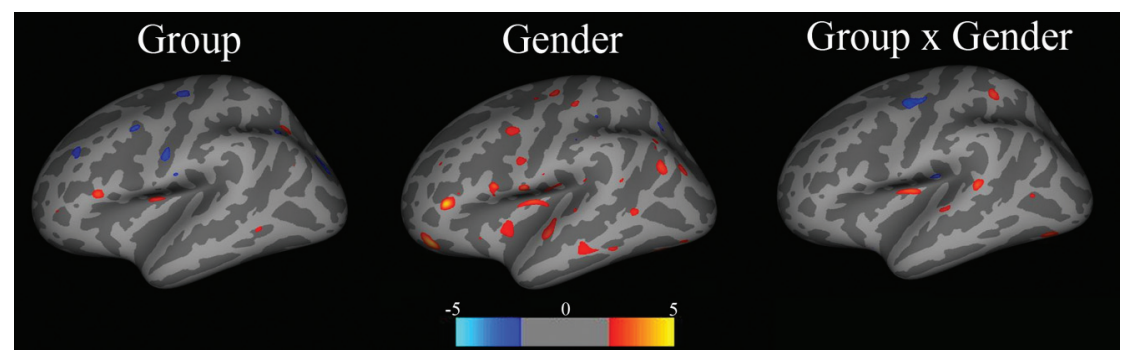

FIG 2. Maps show main effects of group, sex, and group-by-sex interactions for cortical volume. Group: red $=$ control $>$ SDI; blue $=$ SDI $>$ control; Sex: red $=$ male $>$ female, blue $=$ female $>$ male; Group $\times$ Sex: red $=$ female control $>$ female SDI, male control $<$ male SDI. Color bar represents the $z$ score.

pial surface. Many studies showing reduced prefrontal cortex volumes in SDI are based on VBM. ${ }^{3,13,16,37}$ Some investigators using VBM have failed to replicate the finding of reduced gray matter volume in stimulant or polydrug users. ${ }^{18,19}$ Another possibility is that alterations in prefrontal morphometry may have resolved due to the long abstinence, given the evidence that structural and metabolic changes are partially reversible. ${ }^{36,37}$

Although the OFC did not differ between groups, there was a main effect of sex on the OFC. The OFC was larger and thicker in men than in women. A study of 176 healthy controls showed that men had thicker cortices than women in the left orbitofrontal region. ${ }^{26}$ This study and others report that women have thicker parietal and temporal cortices than men. ${ }^{26,44}$ Sexual dimorphism on the brain is not apparent at a gross level, but sex differences in dendritic morphometry, attenuation, and volume have been observed in animals and postmortem brains, emphasizing the importance of modeling sex in cortical thickness investigations.

Thicker and larger OFCs correlated with better negative-reinforcement learning in controls. Reinforcement learning requires adjustments of one's actions on the basis of feedback and is necessary for optimal decision-making. There is evidence that the OFC is in-
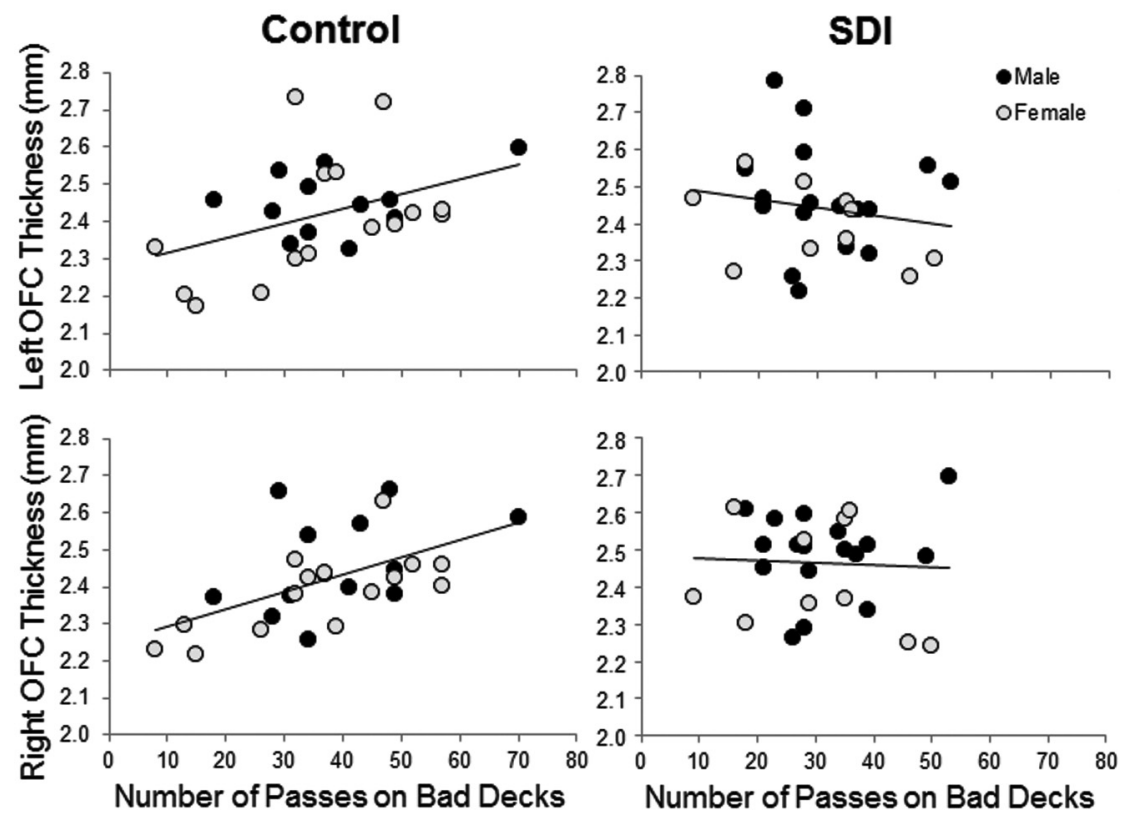

FIG 3. Scatterplots demonstrating correlations between negative-reinforcement learning and OFC thickness. volved in reinforcement learning based on positive feedback. ${ }^{4,45}$ Less is known about the neural correlates of reinforcement learning based on negative feedback, though there is evidence that OFC neurons encode aversive stimuli on the same general scale as rewarding stimuli, suggesting that value information converges in the $\mathrm{OFC}^{46}$ The current findings are consistent with those in a prior study linking prefrontal cortex volume to avoidance learning. ${ }^{16}$ While these preliminary data suggest that the OFC may be involved in learning to avoid loss, interpretation must be tempered by the fact that the correlation was limited to controls.

Significant differences in region-of-interest volume and thickness did not persist after multiple-comparison correction but were verified as trends on whole-brain analyses (Fig 2). Our interpretation is that changes in SDI are small in magnitude and spread over relatively large areas (ie, parcellation unit) as opposed to being large in magnitude in small foci. Such results reflect the high sensitivity of FreeSurfer, which can detect differences in cortical thickness as small as $0.2 \mathrm{~mm}$ with relatively small sample sizes. ${ }^{47} \mathrm{In}$ this article, we focus on 2 frontal and 1 limbic region of interest. While these cortical regions may have specific behavioral correlates in addiction, they are a subset of a larger complex frontostriatallimbic system underlying addictive behavior. Furthermore, we examined only cortical morphometry but recognize that the integrity of this reward system relies on connections between cortical regions.

Limitations of this study are a modest sample size, an inability to determine causality, and an inability to isolate the effects of a single drug. Tobacco and alcohol use is common among stimulant-dependent SDI. A subsample analysis comparing 16 SDI with alcohol dependence to 12 SDI without alcohol dependence revealed no differences in cortical thickness. We cannot exclude the possibility that other drugs influenced the findings. Seven of 28 controls used nicotine, which may have reduced our sensitivity. We did not assess post-traumatic stress disorder and anxiety disorder, which could also influence morphometry.

\section{CONCLUSIONS}

Insula morphology differed in SDI compared with controls, and those differences were modulated by sex. Controls were better than SDI at decision-making involving negative reinforcers. Task performance correlated with OFC measurements in controls. Our findings underscore the importance of accounting for sex in brain morphometry studies. These preliminary results are consistent with the hypothesis that frontolimbic systems may be involved in substance dependence. 


\section{ACKNOWLEDGMENTS}

The authors acknowledge the staff at Addiction Research Treatment Services, Debra Singel, RT, and Amanda Klenk, BS.

Disclosures: Jody Tanabe—RELATED: Grant: National Institutes of Health, ${ }^{*}$ Comments: National Institute on Drug Abuse (NIDA) 024104 and 027748. Manish Dalwani-RELATED: Grant: National Institute on Drug Abuse (DA 009842, DA 011015), ${ }^{*}$ Kane Family Foundation. * Joseph T. Sakai-RELATED: Grant: NIDA, * UNRELATED: Other: Dr Sakai received reimbursement in 2012 for completing a policy review for the WellPoint Office of Medical Policy and Technology Assessment, WellPoint Inc, Thousand Oaks, California. Susan K. Mikulich-Gilbertson-RELATED: Grant: NIDA grants, ${ }^{*}$ Comments: Grant money is paid to the University (Institution), and the University pays my salary proportionately from each grant. Laetitia Thompson—RELATED: Grant: NIDA 024104 and $027748^{*}$ Payment for Writing or Reviewing the Manuscript: NIDA.* Marie Banish—RELATED: Grant: NIDA 027748 * Comments: 1 R01 DA027748. Theodore Krmpotich—RELATED: Grant NIDA 024104 and 027748 . * Money paid to the institution.

\section{REFERENCES}

1. Volkow ND, Fowler JS. Addiction, a disease of compulsion and drive: involvement of the orbitofrontal cortex. Cereb Cortex 2000; 10:318-25

2. Volkow ND, Li TK. Drug addiction: the neurobiology of behaviour gone awry. Nat Rev Neurosci 2004;5:963-70

3. Dom G, Sabbe B, Hulstijn W, et al. Substance use disorders and the orbitofrontal cortex: systematic review of behavioural decisionmaking and neuroimaging studies. Br J Psychiatry 2005;187:209-20

4. Schoenbaum G, Roesch MR, Stalnaker TA. Orbitofrontal cortex, decision-making and drug addiction. Trends Neurosci 2006;29:116-24

5. Naqvi NH, Rudrauf D, Damasio H, et al. Damage to the insula disrupts addiction to cigarette smoking. Science 2007;315:531-34

6. Contreras M, Ceric F, Torrealba F. Inactivation of the interoceptive insula disrupts drug craving and malaise induced by lithium. Science 2007;318:655-58

7. Paulus MP, Stein MB. An insular view of anxiety. Biol Psychiatry 2006;60:383-87

8. Samanez-Larkin GR, Hollon NG, Carstensen LL, et al. Individual differences in insular sensitivity during loss anticipation predict avoidance learning. Psychol Sci 2008;19:320-23

9. Critchley HD. The human cortex responds to an interoceptive challenge. Proc Natl Acad Sci U S A 2004;101:6333-34

10. Gloria R, Angelos L, Schaefer HS, et al. An fMRI investigation of the impact of withdrawal on regional brain activity during nicotine anticipation. Psychophysiology 2009;46:681-93

11. Naqvi NH, Bechara A. The hidden island of addiction: the insula. Trends Neurosci 2009;32:56-67

12. Liu X, Matochik JA, Cadet JL, et al. Smaller volume of prefrontal lobe in polysubstance abusers: a magnetic resonance imaging study. Neuropsychopharmacology 1998;18:243-52

13. Franklin TR, Acton PD, Maldjian JA, et al. Decreased gray matter concentration in the insular, orbitofrontal, cingulate, and temporal cortices of cocaine patients. Biol Psychiatry 2002;51:134-42

14. Matochik JA, London ED, Eldreth DA, et al. Frontal cortical tissue composition in abstinent cocaine abusers: a magnetic resonance imaging study. Neuroimage 2003;19:1095-102

15. Lyoo IK, Pollack MH, Silveri MM, et al. Prefrontal and temporal gray matter density decreases in opiate dependence. Psychopharmacology (Berl) 2006;184:139-44

16. Tanabe J, Tregellas JR, Dalwani M, et al. Medial orbitofrontal cortex gray matter is reduced in abstinent substance-dependent individuals. Biol Psychiatry 2009;65:160-64

17. Thompson PM, Hayashi KM, Simon SL, et al. Structural abnormalities in the brains of human subjects who use methamphetamine. J Neurosci 2004;24:6028-36

18. Schlaepfer TE, Lancaster E, Heidbreder R, et al. Decreased frontal white-matter volume in chronic substance abuse. Int J Neuropsychopharmacol 2006;9:147-53

19. Narayana PA, Datta S, Tao G, et al. Effect of cocaine on structural changes in brain: MRI volumetry using tensor-based morphometry. Drug Alcohol Depend 2010;111:191-99

20. Fischl B, Dale AM. Measuring the thickness of the human cerebral cortex from magnetic resonance images. Proc Natl Acad Sci U S A 2000;97:11050-55

21. Makris N, Gasic GP, Kennedy DN, et al. Cortical thickness abnormalities in cocaine addiction: a reflection of both drug use and a pre-existing disposition to drug abuse? Neuron 2008;60:174-88
22. Durazzo TC, Tosun D, Buckley S, et al. Cortical thickness, surface area, and volume of the brain reward system in alcohol dependence: relationships to relapse and extended abstinence. Alcohol Clin Exp Res 2011;35:1187-200

23. Kühn S, Schubert F, Gallinat J. Reduced thickness of medial orbitofrontal cortex in smokers. Biol Psychiatry 2010;68:1061-65

24. Greenfield SF, Back SE, Lawson K, et al. Substance abuse in women Psychiatr Clin North Am 2010;33:339-55

25. Volkow ND, Tomasi D, Wang GJ, et al. Reduced metabolism in brain "control networks" following cocaine-cues exposure in female cocaine abusers. PLoS One 2011;6:e16573

26. Sowell ER, Peterson BS, Kan E, et al. Sex differences in cortical thickness mapped in 176 healthy individuals between 7 and 87 years of age. Cereb Cortex 2007;17:1550-60

27. Medina KL, McQueeny T, Nagel BJ, et al. Prefrontal cortex volumes in adolescents with alcohol use disorders: unique gender effects. Alcohol Clin Exp Res 2008;32:386-94

28. Thompson LL, Claus ED, Mikulich-Gilbertson SK, et al. Negative reinforcement learning is affected in substance dependence. Drug Alcohol Depend 2012;123:84-90

29. Bechara A, Damasio AR, Damasio H, et al. Insensitivity to future consequences following damage to human prefrontal cortex. Cognition 1994;50:7-15

30. Bechara A, Dolan S, Denburg N, et al. Decision-making deficits, linked to a dysfunctional ventromedial prefrontal cortex, revealed in alcohol and stimulant abusers. Neuropsychologia 2001;39:376-89

31. Petry NM. Substance abuse, pathological gambling, and impulsiveness. Drug Alcohol Depend 2001;63:29-38

32. Desikan RS, Segonne F, Fischl B, et al. An automated labeling system for subdividing the human cerebral cortex on MRI scans into gyral based regions of interest. Neuroimage 2006;31:968-80

33. Cohen J. Statistical Power Analysis for the Behavioral Sciences. 2nd ed. Hillsdale, New Jersey: Lawrence Erlbaum Associates; 1988

34. Lopez-Larson MP, Bogorodzki P, Rogowska J, et al. Altered prefrontal and insular cortical thickness in adolescent marijuana users. Behav Brain Res 2011;220:164-72

35. Schwartz DL, Mitchell AD, Lahna DL, et al. Global and local morphometric differences in recently abstinent methamphetaminedependent individuals. Neuroimage 2010;50:1392-401

36. Gazdzinski S, Durazzo TC, Meyerhoff DJ. Temporal dynamics and determinants of whole brain tissue volume changes during recovery from alcohol dependence. Drug Alcohol Depend 2005;78:263-73

37. Kim SJ, Lyoo IK, Hwang J, et al. Prefrontal grey-matter changes in short-term and long-term abstinent methamphetamine abusers. Int J Neuropsychopharmacol 2006;9:221-28

38. Paulus MP, Rogalsky C, Simmons A, et al. Increased activation in the right insula during risk-taking decision making is related to harm avoidance and neuroticism. Neuroimage 2003;19:1439-48

39. Paulus MP, Tapert SF, Schuckit MA. Neural activation patterns of methamphetamine-dependent subjects during decision making predict relapse. Arch Gen Psychiatry 2005;62:761-68

40. Potenza MN, Hong KI, Lacadie CM, et al. Neural correlates of stressinduced and cue-induced drug craving: influences of sex and cocaine dependence. Am J Psychiatry 2012;169:406-14

41. London ED, Ernst M, Grant S, et al. Orbitofrontal cortex and human drug abuse: functional imaging. Cereb Cortex 2000;10:334-42

42. Lawyer G, Bjerkan PS, Hammarberg A, et al. Amphetamine dependence and co-morbid alcohol abuse: associations to brain cortical thickness. BMC Pharmacol 2010;10:5

43. Wonderlick JS, Ziegler DA, Hosseini-Varnamkhasti P, et al. Reliability of MRI-derived cortical and subcortical morphometric measures: effects of pulse sequence, voxel geometry, and parallel imaging. Neuroimage 2009;44:1324-33

44. Im K, Lee JM, Lee J, et al. Gender difference analysis of cortical thickness in healthy young adults with surface-based methods. Neuroimage 2006;31:31-38

45. Roesch MR, Olson CR. Neuronal activity related to reward value and motivation in primate frontal cortex. Science 2004;304: 307-10

46. Morrison SE, Salzman CD. The convergence of information about rewarding and aversive stimuli in single neurons. J Neurosci 2009;29:11471-83

47. Han X, Jovicich J, Salat D, et al. Reliability of MRI-derived measurements of human cerebral cortical thickness: the effects of field strength, scanner upgrade and manufacturer. Neuroimage 2006;32: $180-94$ 